\title{
STUDY OF LOW-SPIN STATES IN Ca ISOTOPES VIA NEUTRON CAPTURE REACTIONS*
}



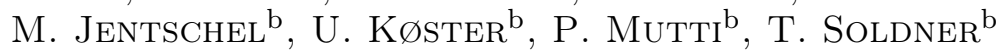

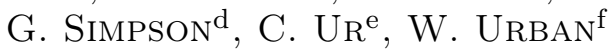 \\ ${ }^{a}$ Università degli Studi di Milano and INFN Sez. Milano, Italy \\ ${ }^{\mathrm{b}}$ ILL, 71 Avenue des Martyrs, 38042 Grenoble CEDEX 9, France \\ ${ }^{\mathrm{c}}$ GANIL, BP 55027, 14076 Caen CEDEX 5, France \\ ${ }^{\mathrm{d}}$ LPSC, 53 Avenue des Martyrs, 38026 Grenoble, France \\ ${ }^{\mathrm{e}}$ INFN Sez. Padova, Italy and ELI-NP Bucharest-Magurele, Romania \\ ${ }^{\mathrm{f}}$ Faculty of Physics, Univ. of Warsaw, Hoża 69, 00-681 Warszawa, Poland
}

(Received March 23, 2015)

Preliminary results are presented from a $\gamma$-spectroscopy study of lowspin states of ${ }^{41,49} \mathrm{Ca}$ isotopes, produced by neutron capture on a Ca target, at very high coincidence rates. The experiment was performed at the PF1B cold-neutron facility at ILL (Grenoble, France), using the EXILL array, consisting of EXOGAM, GASP and ILL-CLOVER detectors.

DOI:10.5506/APhysPolB.46.647

PACS numbers: 23.20.En, 23.20.Lv, 27.40. $+\mathrm{z}, 28.20 . \mathrm{Np}$

\section{Introduction}

One of the main issues in nuclear physics concerns the interplay between single particle and collective degrees of freedom, which is a manifestation of the many body nature of the atomic nucleus. Nuclei around doubly closed shells offer an excellent testing ground to investigate such a phenomenon, particularly in terms of couplings between core excitations (i.e. phonons) and single particles outside the closed shell core [1]. This type of couplings are at the origin of the anharmonicities of vibrational spectra, damping phenomena, quenching of spectroscopic factors, ...

The coupling between a core vibration (phonon of multipolarity $\lambda$ ) and a single particle with spin $j$ generates a multiplet of states that are characterized by a well defined parity and by angular momenta between $|\lambda-j|$ and

* Presented at the Zakopane Conference on Nuclear Physics "Extremes of the Nuclear Landscape", Zakopane, Poland, August 31-September 7, 2014. 
$\lambda+j$. At the leading order, the coupling is linear with the vibration, therefore its strength can be evaluated by measuring the transition probability to the ground state.

From the experimental point of view, indications of particle-phonon coupled states have been found mostly in medium-heavy nuclei [1], while scarce information exists for medium-light systems [2-5] and moving away from the stability valley. In the latter case, particle-phonon coupled states would also provide complementary information on the robustness or softness of the nuclear collectivity far from stability.

In this context, the doubly magic ${ }^{48} \mathrm{Ca}$ is particularly interesting. In the work by Montanari et al. [2, 3] (an in-beam $\gamma$-spectroscopy study of $n$-rich nuclei around ${ }^{48} \mathrm{Ca}$ populated the ${ }^{48} \mathrm{Ca}+{ }^{64} \mathrm{Ni}$ binary reaction, at $\approx$ $6 \mathrm{MeV} / u$ ), it was demonstrated that the $9 / 2^{+}$level of ${ }^{49} \mathrm{Ca}($ at $4.017 \mathrm{MeV}$ ) is a member of the multiplet of states $\left(3 / 2^{+}, 5 / 2^{+}, 7 / 2^{+}\right.$and $\left.9 / 2^{+}\right)$arising by coupling the $3^{-}$octupole phonon of ${ }^{48} \mathrm{Ca}$ with the unpaired $p_{3 / 2}$ neutron. To obtain a complete picture of particle-phonon coupled states in the ${ }^{49} \mathrm{Ca}$ nucleus, one should also study the other members of the $p_{3 / 2} \otimes 3^{-}$multiplet, employing a reaction mechanism which allows to populate them with sizable cross section. In this contribution, we report preliminary results from a neutron capture experiment on a ${ }^{48} \mathrm{Ca}$ target, aiming at a high-resolution $\gamma$-spectroscopy of low-spin states in ${ }^{49} \mathrm{Ca}$, from the capture level to the ground state.

\section{Experimental setup and data analysis}

The experiment was performed at the PF1B cold-neutron facility at the Istitute Laue Langevine (Grenoble, France), using the EXILL array, consisting of 8 EXOGAM clovers, 6 large coaxial detectors from GASP and 2 ILL-CLOVERS, placed at $90^{\circ}, 45^{\circ}$ and $0^{\circ}$ with respect to the beam direction (the latter at $180^{\circ}$ with respect to the GASP detectors), respectively. The total photopeak efficiency was about $6 \%$. A digital data acquisition allowed event rates up to $0.84 \mathrm{MHz}$ to be handled [6].

The $(n, \gamma)$ reaction was performed using a $620 \mathrm{mg} \mathrm{CaCO}_{3}$ target with $69.2 \%, 27.9 \%$ and $2.5 \%$ istopic abundances of ${ }^{48} \mathrm{Ca},{ }^{40} \mathrm{Ca}$ and ${ }^{44} \mathrm{Ca}$, respectively. As a consequence, a large fraction of double and triple $\gamma$ coincidences were coming from ${ }^{41} \mathrm{Ca},{ }^{45} \mathrm{Ca}$ and from ${ }^{49} \mathrm{Sc}$, populated by $\beta$-decay of ${ }^{49} \mathrm{Ca}$. In general, the populated nuclei of ${ }^{41,45,49} \mathrm{Ca}$ were found to decay from the capture level by primary (E1) transitions of several $\mathrm{MeV}$ and to populate in a statistical way, excited states within few units of spin. By using several cascades, the neutron separation energies were calculated to be 8363.10(42), 7414.34(35) and 5146.46(50) keV for ${ }^{41} \mathrm{Ca},{ }^{45} \mathrm{Ca}$ and ${ }^{49} \mathrm{Ca}$, respectively. Such values agree, within the errors, with the ones reported in literature [7]. 
Figure 1 (a) shows the total spectrum measured in the $n$-capture reaction, with strong lines coming from the de-excitation of ${ }^{41,45,49} \mathrm{Ca}$ and ${ }^{49} \mathrm{Sc}$. A preliminary level scheme of ${ }^{49} \mathrm{Ca}$ is given in panel (b), with newly found transitions marked in grey (red). As a consequence of the low energy of the $1 / 2^{+}$capture level in ${ }^{49} \mathrm{Ca}$, only two-steps cascades are observed, with intermediate states of negative parity. On the contrary, in ${ }^{41} \mathrm{Ca}$ and ${ }^{45} \mathrm{Ca}$ multisteps cascades were observed, populating intermediate states of both positive and negative parities. In particular, several positive parity states are found in 3-4 MeV region where one expects to find states of particlephonon nature, based on the coupling between the $p_{3 / 2}$ neutron and the $3^{-}$ octupole phonon of ${ }^{40} \mathrm{Ca}$ and ${ }^{44} \mathrm{Ca}$, at 3.7 and $3.3 \mathrm{MeV}$, respectively.
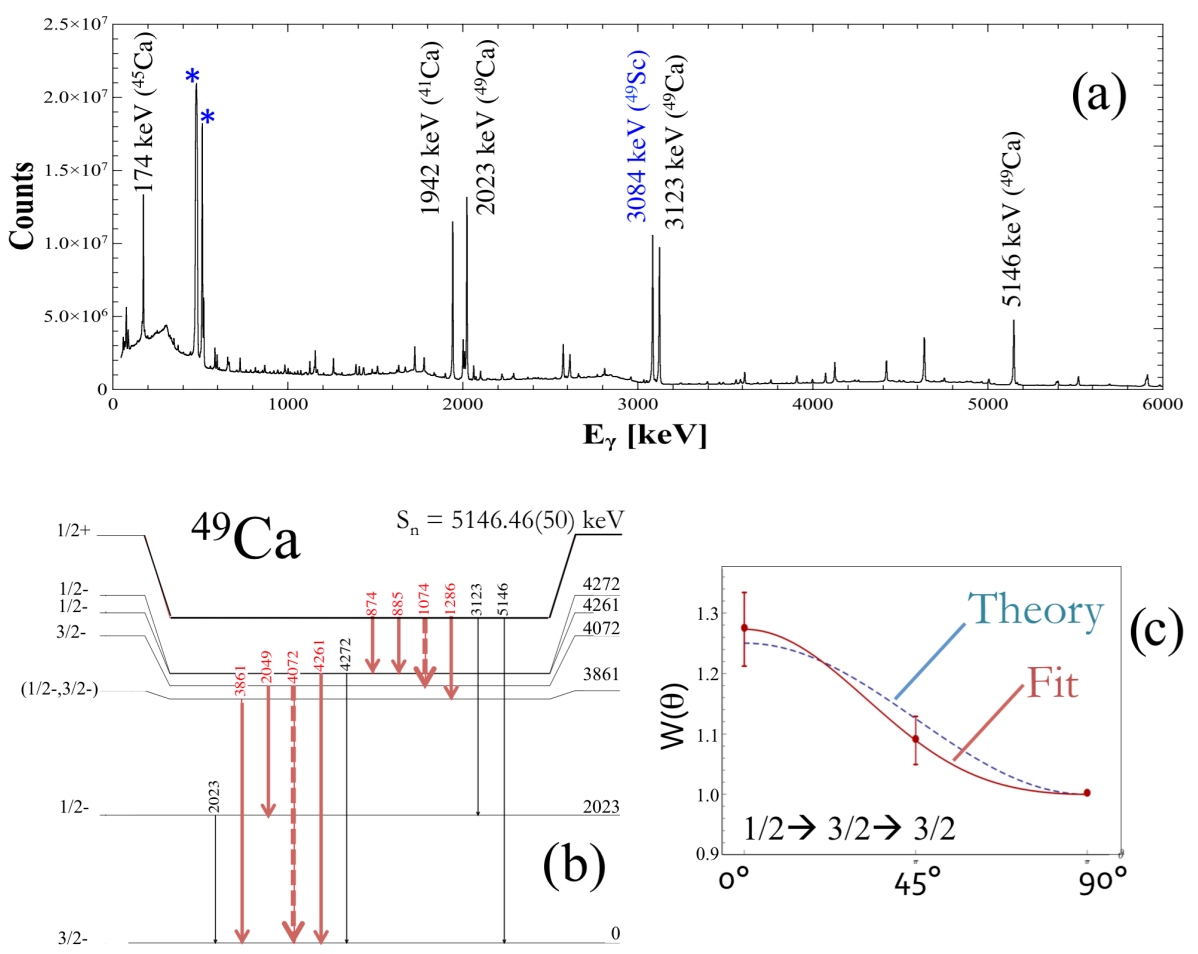

Fig. 1. (a) Total $\gamma$ spectrum measured in the $n$-capture reaction with the $\mathrm{Ca}$ target. The strongest transitions from ${ }^{41,45,49} \mathrm{Ca}$ and ${ }^{49} \mathrm{Sc}$ are given. Background lines are marked by stars. (b) Preliminary level scheme of ${ }^{49} \mathrm{Ca}$ with newly found transitions given in grey (red). (c) Angular correlation of the 1074 and $4072 \mathrm{keV}$ transitions (dotted arrows in (b)). Solid and dashed lines are the experimental fit and the theoretical interpolation (obtained by fitting the $\delta$ mixing parameter), giving the spin assignment $1 / 2,3 / 2$ and $3 / 2$, for the initial, middle and final states, respectively. 
A key aspect of this work is the measurements of $\gamma-\gamma$ angular correlations, performed with the EXOGAM clovers, to firmly establish the spin of several excited states. The symmetry of the EXOGAM detectors allowed angular correlation measurements at three relative angles between the detectors, i.e. $0^{\circ}, 45^{\circ}$ and $90^{\circ}$ degrees. The analysis was performed following the formalism of Krane et al. [8], adopting their convention on the sign for the mixing coefficient $\delta$. The expression for the angular correlation function between two consecutive $\gamma$ transitions, $\gamma_{1}$ and $\gamma_{2}$ (in a cascade emitted from an unoriented state with spin $J_{1}$, through an intermediate level with spin $J_{2}$ to the final level with spin $J_{3}$ ) can be expanded into a series of Legendre polynomials $P_{k}$ of rank $k$

$$
W(\theta)=1+a_{22} q_{2} P_{2}(\cos (\theta))+a_{44} q_{4} P_{4}(\cos (\theta)),
$$

where $\theta$ is the angle between the directions of $\gamma_{1}$ and $\gamma_{2}$, while $q_{2}$ and $q_{4}$ are the attenuation coefficients taking into account the finite geometry of the apparatus. Figure 1 (c) shows the angular correlation obtained for the 1074 and $4072 \mathrm{keV}$ transitions of ${ }^{49} \mathrm{Ca}$. The spin of the initial, intermediate and final states are confirmed to be $1 / 2,3 / 2$ and $3 / 2$, respectively, taking the $4073 \mathrm{keV}$ line as a pure dipole and a mixing $\delta$ on the $1074 \mathrm{keV}$ transition equal to $-1.87_{-0.22}^{+0.19}$, which indicates a strong dipole character.

\section{Conclusions}

Preliminary results were presented from a neutron capture experiment performed at ILL (Grenoble) on a Ca target. The setup consisted of the high-efficiency Ge array EXILL, which allowed to perform high-resolution $\gamma$-spectroscopy and angular correlation studies of ${ }^{41,45,49} \mathrm{Ca}$ nuclei. The work aims, in particular, at identifying states arising by coupling single particles to core excitations, such as the $3^{-}$octupole phonon in the corresponding ${ }^{40,44,48} \mathrm{Ca}$ cores.

\section{REFERENCES}

[1] A. Bohr, B.R. Mottelson, Nuclear Structure, I and II, W.A. Benjamin, 1975.

[2] D. Montanari et al., Phys. Lett. B697, 288 (2011).

[3] D. Montanari et al., Phys. Rev. C85, 044301 (2012).

[4] G. Bocchi et al., Phys. Rev. C89, 054302 (2014).

[5] C.R. Niţă et al., Phys. Rev. C89, 064314 (2014).

[6] G. De France et al., Eur. Phys. J. Web Conf. 66, 02010 (2014).

[7] National Nuclear Data Center; http://www.nndc.bnl.gov/nudat2/

[8] K.S. Krane et al., At. Data Nucl. Data Tables 11, 351 (1973). 